\title{
A simple proof of asymptotic consensus in the Hegselmann-Krause and Cucker-Smale models with normalization and delay
}

\author{
Jan Haskovec*
}

\begin{abstract}
We present a simple proof of asymptotic consensus in the discrete Hegselmann-Krause model and flocking in the discrete Cucker-Smale model with normalization and variable delay. It utilizes the convexity of the normalized communication weights and a Gronwall-Halanay-type inequality. The main advantage of our method, compared to previous approaches to the delay Hegselmann-Krause model, is that it does not require any restriction on the maximal time delay, or the initial data, or decay rate of the influence function. From this point of view the result is optimal. For the Cucker-Smale model it provides an analogous result in the regime of unconditonal flocking with sufficiently slowly decaying communication rate, but still without any restriction on the length of the maximal time delay. Moreover, we demonstrate that the method can be easily extended to the mean-field limits of both the HegselmannKrause and Cucker-Smale systems, using appropriate stability results on the measure-valued solutions.
\end{abstract}

Keywords: Hegselmann-Krause model, asymptotic consensus, Cucker-Smale model, flocking, long-time behavior, variable delay.

\section{Introduction}

In this paper we study asymptotic behavior of the Hegselmann-Krause [13] and Cucker-Smale [5, 6] models with normalized communication weights and variable time delay. The Hegselmann-Krause model describes the evolution of $N \in \mathbb{N}$ agents who adapt their opinions to the ones of other members of the group. Agent $i$ 's opinion is represented by the quantity $x_{i}=x_{i}(t) \in \mathbb{R}^{d}$, with $d \in \mathbb{N}$ the space dimension, which is a function of time $t \geq 0$. For many applications in biological and socio-economical systems or control problems (for instance, swarm robotics $[11,23])$, it is natural to include a time delay in the model reflecting the time needed for each agent to receive information from other agents. We therefore assume that agents' communication takes place subject to a variable delay $\tau=\tau(t) \geq 0$, i.e., agent $i$ with opinion $x_{i}(t)$ receives at time $t>0$ the information about the opinion of agent $j$ in the form $x_{j}(t-\tau(t))$. The opinions evolve then according to the following Hegselmann-Krause-type dynamics,

$$
\dot{x}_{i}(t)=\sum_{j \neq i} \psi_{i j}(t)\left(x_{j}(t-\tau(t))-x_{i}(t)\right), \quad i=1, \ldots, N .
$$

The communication weights $\psi_{i j}=\psi_{i j}(t)$ measure the intensity of the influence between agents depending on the dissimilarity of their opinions. In this paper we consider the normalized weights introduced in [18],

$$
\psi_{i j}(t):= \begin{cases}\frac{\psi\left(\left|x_{j}(t-\tau(t))-x_{i}(t)\right|\right)}{\sum_{\ell \neq i} \psi\left(\left|x_{\ell}(t-\tau(t))-x_{i}(t)\right|\right)} & \text { if } j \neq i, \\ 0 & \text { if } j=i,\end{cases}
$$

\footnotetext{
*Computer, Electrical and Mathematical Sciences \& Engineering, King Abdullah University of Science and Technology, 23955 Thuwal, KSA. jan.haskovec@kaust.edu.sa
} 
where the nonnegative influence function $\psi:[0, \infty) \rightarrow[0, \infty)$, also called communication rate, measures how strongly each agent is influenced by others depending on their distance (dissimilarity of their opinions). The normalization in (2) scales the communication weights relative to the influence of all other agents without involving explicit dependence on their number. As discussed in [18], the "classical" model where the influence is scaled by $1 / N$ has the drawback that the dynamics of an agent is modified by the total number of agents even if its dynamics is only significantly influenced by a few nearby agents. The normalization removes this drawback, but still allows for a meaningful passage to the mean field limit $N \rightarrow \infty$. However, it destroys the symmetry of the weights, i.e., in general, $\psi_{i j} \neq \psi_{j i}$, which makes the system more challenging for analysis.

The variable time delay $\tau=\tau(t)$ is assumed to be nonegative and uniformly bounded by some $\bar{\tau}>0$; this includes the generic case of the constant delay $\tau(t) \equiv \bar{\tau}$. The system (1) is equipped with the initial datum

$$
x_{i}(s)=x_{i}^{0}(s), \quad i=1, \cdots, N, \quad s \in[-\bar{\tau}, 0],
$$

with prescribed trajectories $x_{i}^{0} \in C([-\bar{\tau}, 0]), i=1, \cdots, N$.

The phenomenon of consensus finding in the context of (1) refers to the (asymptotic) emergence of one or more opinion clusters formed by agents with (almost) identical opinions [15]. Global consensus is the state where all agents have the same opinion, i.e., $x_{i}=x_{j}$ for all $i, j \in\{1, \ldots, N\}$. Convergence to global consensus as $t \rightarrow \infty$ for the system (1) has been proved in [4] under a set of conditions requiring smallness of the maximal time delay in relation to the decay speed of the influence function and the fluctuation of the initial datum. However, as we shall discuss in Section 2, there is justified expectation that asymptotic global consensus is reached without any restriction on the maximal time delay $\bar{\tau}$, for all initial data, and only assuming that the influence function is strictly positive on $[0, \infty)$, but may decay to zero arbitrarily fast at infinity. In this paper we prove that this intuitive expectation is indeed true. As this result is essentially optimal, it closes an important gap in the theory of asymptotic behavior of the Hegselmann-Krause model with delay.

Our method of proof consists of three steps. First, we prove nonexpansivity of the agent group, i.e., a uniform bound on the position radius of the agents. Second, we apply a geometric argument, exploiting the stochasticity property of the normalized weights (2),

$$
\sum_{j \neq i} \psi_{i j}=1 \quad \text { for all } i=1, \cdots, N .
$$

This implies that the terms $\sum_{j=1}^{N} \psi_{i j}(t) x_{j}(t-\tau(t))$ in (1) are convex combinations of the vectors $x_{j}(t-\tau(t))$ and provides an upper estimate on their distance in terms of a lower estimate on the communication weights. This facilitates a derivation of a delay differential inequality on the spatial diameter of the group, which in the third step is shown to decay exponentially to zero by employing a Gronwall-Halanay-type inequality. In particular, we avoid the need to work with Lyapunov-Krasovskii-type functionals, despite the presence of the delay and the broken symmetry $\psi_{i j} \neq \psi_{j i}$.

A second goal of this paper is to study, by a slight extension of the above method, the asymptotic behavior of a variant of the Cucker-Smale model $[5,6]$ with normalized communication weights, as introduced by Motsch and Tadmor [18]. The model can be seen as a second-order version of (1) and reads, for $i=1, \cdots, N$,

$$
\begin{aligned}
& \dot{x}_{i}(t)=v_{i}(t), \\
& \dot{v}_{i}(t)=\sum_{j \neq i} \psi_{i j}(t)\left(v_{j}(t-\tau(t))-v_{i}(t)\right),
\end{aligned}
$$

where the normalized communication weights $\psi_{i j}=\psi_{i j}(t)$ are again given by (2). The system is equipped with the initial datum

$$
x_{i}(s)=x_{i}^{0}(s), \quad v_{i}(s)=v_{i}^{0}(s), \quad i=1, \cdots, N, \quad s \in[-\bar{\tau}, 0],
$$


with prescribed phase-space trajectories $x_{i}^{0}, v_{i}^{0} \in C\left([-\bar{\tau}, 0] ; \mathbb{R}^{d}\right), i=1, \cdots, N$. For physical reasons it may be required that

$$
x_{i}^{0}(s)=x_{i}^{0}(-\bar{\tau})+\int_{-\bar{\tau}}^{s} v_{i}^{0}(\sigma) \mathrm{d} \sigma \quad \text { for } s \in(-\bar{\tau}, 0],
$$

but we do not pose this particular restriction here.

The Cucker-Smale model (and its variants) is in the literature considered a generic model for flocking, herding or schooling of animals with positions $x_{i}=x_{i}(t)$ and velocities $v_{i}=v_{i}(t)$. If the influence function has a heavy tail, i.e., $\int^{\infty} \psi(s)=\infty$, then the model exhibits the so-called unconditional flocking, where (13) holds for every initial configuration, see $[5,6,10,9]$. In the opposite case the flocking is conditional, i.e., the asymptotic behavior of the system depends on the initial configuration. Cucker-Smale-type models with delay were studied in $[17,8,12,2,3]$. In particular, in [17] and [2] the precise form (5) with the normalized communication rates (2) was considered (with constant time delay) and asymptotic flocking was proved under a smallness condition on the delay, related to the decay properties of the influence function $\psi$ and the velocity diameter of the initial datum. In [17] the authors generalize the technique of estimating "maximal action of antisymmetric matrices", developed in [18], to the case with delay. However, this approach does not benefit from the convexity property of the normalized communication weights and, therefore, leads to suboptimal results. The method of proof in [2] is more direct, based on deriving a system of dissipative delay differential inequalities.

The main benefit of this paper is that it offers a significantly simpler and more intuitive idea of proof of the flocking behavior, which optimally exploits the convexity property of the normalized weights (4) and avoids the use of Lyapunov-Krasovskii-type functionals. The convexity argument, embodied in Lemma 2 below, is an adaptation of the notion of coefficient of ergodicity introduced by Dobrushin [7] in the context of Markov chains. It was later used to quantify the contractivity in first-order, discrete-time models of opinion dynamics (without delay) in [16]. In [14] the class of scrambling matrices was introduced, which can be characterized as stochastic matrices of communication weights with sufficient connectivity between agents. Then, the convexity argument, exploiting the stochasticity of the matrix, is used to provide a bound on the contractivity of the system, which ultimately leads to a proof of asymptotic consensus. A similar argument was applied to second-order models (without delay) in [19]. The main novelty of our paper is the adaptation of the convexity argument to both the first- and second-order consensus systems with delay, and its combination with the Gronwall-Halanay-type inequality that directly provides exponential decay of the spatial diameter of the group. In contrast to [14] we consider strictly positive communication weights, i.e., there is a direct communication link between every pair of agents. This simplifies the analysis since the notion of scrambling matrices is not necessary (in other words, our matrix of communication weights is scrambling by definition).

Models of consensus finding and flocking with delay have been extensively studied in the Engineering community, with results going back to [20]. For linear problems, where the communication weights $\psi_{i j}$ are fixed, stability criteria based on the frequency approach and on Lyapunov-Krasovskii techniques were derived in [21]. We note that although our approach focuses on the nonlinear setting with $\psi_{i j}$ given by (2), it of course does apply also the the linear case, as long as the weights verify the convexity property (4). A simple and elegant proof of delay-independent consensus and flocking in nonlinear networks with multiple time-varying delays under very mild assumptions was provided in [24, 25]. This approach is based on $\ell^{\infty}$-estimates obtained using fundamental concepts from the non-negative matrix theory and a LyapunovKrasovskii functional. Our method is conceptually different (and, arguably, even simpler), based on the geometric convexity argument of Lemma 2 and avoiding the need to apply the techniques of non-negative matrix theory. Also, instead of working with a Lyapunov-Krasovskii functional, we prove directly the exponential decay of the position/velocity diameters with calculable rate.

The paper is organized as follows. In Section 2 we explain the intuitive motivation for our results, formulate the precise assumptions on the influence function $\psi=\psi(s)$ and delay $\tau=\tau(t)$, and state our main results. Their proofs for the Hegselmann-Krause model (1) are presented in Section 3, and their extension for the Cucker-Smale model (5) in Section 4. Finally, in Section 5 we prove the analogues of the consensus and flocking results for the mean-field limits of (1) and (5). 


\section{Motivation, assumptions and main results}

Let us explain the intuitive expectation that all solutions of (1) should converge to global consensus as $t \rightarrow \infty$, regardless of the length of the delay. For this sake, we consider the case of two agents, $N=2$, with positions $x_{1}(t), x_{2}(t)$, and constant delay $\tau(t) \equiv \tau$. Then (2) gives $\psi_{12}=\psi_{21}=1$ and (1) reduces to the linear system

$$
\begin{aligned}
& \dot{x}_{1}(t)=x_{2}(t-\tau)-x_{1}(t), \\
& \dot{x}_{2}(t)=x_{1}(t-\tau)-x_{2}(t) .
\end{aligned}
$$

Defining $w:=x_{1}-x_{2}$, we have

$$
\dot{w}(t)=-w(t-\tau)-w(t) .
$$

Assuming a solution of the form $w(t)=e^{\xi t}$ for some complex $\xi \in \mathbb{C}$, we obtain the characteristic equation

$$
\xi=-e^{-\xi \tau}-1
$$

A simple inspection reveals that all roots $\xi$ have negative real part, which implies that all solutions $w(t)$ of (7) tend to zero as $t \rightarrow \infty$. I.e., we have the asymptotic consensus $\lim _{t \rightarrow \infty} x_{1}(t)-x_{2}(t)=0$, for any value of the constant delay $\tau$.

Let us note that the situation is fundamentally different for a reaction-type delay in (1), i.e., the system

$$
\dot{x}_{i}(t)=\sum_{j=1}^{N} \psi_{i j}(t)\left(x_{j}(t-\tau(t))-x_{i}(t-\tau(t))\right), \quad i=1, \ldots, N .
$$

Then, considering again $N=2$ and constant delay $\tau(t) \equiv \tau$, we have for $w:=x_{1}-x_{2}$,

$$
\dot{w}(t)=-2 w(t-\tau) .
$$

Nontrivial solutions of this equation exhibit oscillations whenever $2 \tau>e^{-1}$ and the amplitude of the oscillations diverges in time if $2 \tau>\pi / 2$, see, e.g., [22]. The different types of asymptotic behavior of the Hegselmann-Krause system with communication-type delay (1) versus the system with reaction-type delay (8) can be intuitively understood by noting that the instantaneous negative feedback term $-x_{i}(t)$ has a stabilizing effect, while the delay terms typically destabilize, and this effect becomes stronger with longer delays. Thus, the stabilizing effect of the instantaneous term $-x_{i}(t)$ in the right-hand side of $(1)$ is stronger than the destabilizing effect of the delay terms $x_{j}(t-\tau(t))$, regardless of the maximal length of the delay $\bar{\tau}$. In (8) the stabilizing effect is not present, and therefore, for large enough delays, the solutions may diverge as $t \rightarrow \infty$.

Let us now formulate the precise assumptions on the influence function and variable delay that we adopt throughout the paper. The influence function $\psi \in C([0, \infty))$ shall satisfy

$$
0<\psi(s) \leq 1 \quad \text { for all } s \geq 0 .
$$

Note that we do not require any monotonicity properties of $\psi$. For the variable delay function $\tau \in C([0, \infty))$ we pose the assumption

$$
0 \leq \tau(t) \leq \bar{\tau} \quad \text { for all } t \geq 0
$$

for some fixed $\bar{\tau}>0$. Clearly, the generic case of constant delay $\tau(t) \equiv \bar{\tau}$ is included in (10). Let us stress that both the above assumptions are very minimal. The global positivity of $\psi$ in (9) cannot be further relaxed, since universal consensus behavior cannot be expected if $\psi$ would be allowed to vanish even pointwise. The upper bound $\psi \leq 1$ can be replaced by any arbitrary positive value due to the scaling invariance of (2). The variable delay $\tau=\tau(t)$ is allowed to vanish on arbitrary subsets of $[0, \infty)$. 
To formulate our main results, let us introduce the spatial diameter $d_{x}=d_{x}(t)$ of the agent group,

$$
d_{x}(t):=\max _{1 \leq i, j \leq N}\left|x_{i}(t)-x_{j}(t)\right|
$$

The global asymptotic consensus is then defined as the property

$$
\lim _{t \rightarrow \infty} d_{x}(t)=0
$$

Let us note that due to the broken symmetry $\psi_{i j} \neq \psi_{j i}$, the system (1) does not conserve the mean value $\frac{1}{N} \sum_{i=1}^{N} x_{i}$. Consequently, the (asymptotic) consensus vector cannot be inferred from the initial datum in a straightforward way and can be seen as an emergent property of the system. This makes, in general, the convergence results more delicate to obtain. However, our convexity-based approach is robust with respect to this issue.

Our main result regarding the consensus behavior of the Hegselmann-Krause model (1), which will be proved in Section 3, is as follows.

Theorem 1. Let $N \geq 3$ and let the assumptions (9) on $\psi=\psi(s)$ and $(10)$ on $\tau=\tau(t)$ be verified. Then all solutions of (1) reach global asymptotic consensus as defined by (12). The decay of $d_{x}=d_{x}(t)$ to zero is exponential with calculable rate that improves with increasing $N$.

For the Cucker-Smale-type system (5), we define asymptotic flocking as the property

$$
\lim _{t \rightarrow \infty} d_{v}(t)=0, \quad \sup _{t \geq 0} d_{x}(t)<\infty,
$$

where the velocity diameter $d_{v}=d_{v}(t)$ of the agent group is given by

$$
d_{v}(t):=\max _{1 \leq i, j \leq N}\left|v_{i}(t)-v_{j}(t)\right|
$$

We also introduce the velocity radius $R_{v}=R_{v}(t)$,

$$
R_{v}(t):=\max _{1 \leq i \leq N}\left|v_{i}(t)\right|
$$

Again, we observe that (5) does not conserve the global momentum $\frac{1}{N} \sum_{i=1}^{N} v_{i}$. Finally, we introduce the quantity

$$
\Psi(r):=\inf _{s \in(0, r)} \psi(s)
$$

The following result will be proved in Section 4 .

Theorem 2. Let $N \geq 3$ and let the assumptions (9) on $\psi=\psi(s)$ and (10) on $\tau=\tau(t)$ be verified. Moreover, assume that there exists $C \in(0,1)$ such that

$$
1-C=\left(1-\frac{N-2}{N-1} \Psi\left(\bar{\tau} R_{v}^{0}+d_{x}^{0}+\frac{d_{v}^{0}}{C}\right)\right) e^{C \bar{\tau}},
$$

with

$$
R_{v}^{0}:=\max _{s \in[-\bar{\tau}, 0]} R_{v}(s), \quad d_{x}^{0}:=\max _{s \in[-\bar{\tau}, 0]} d_{x}(s), \quad d_{v}^{0}:=\max _{s \in[-\bar{\tau}, 0]} d_{v}(s)
$$

Then the solution $(x(t), v(t))$ of (5) subject to the initial datum (6) exhibits asymptotic flocking as defined by (13). Moreover, the decay of the velocity diameter $d_{v}=d_{v}(t)$ to zero as $t \rightarrow \infty$ is exponential with rate C. 
Let us note that the assumption (15), although it may seem technical, is in fact very natural in the context of the Cucker-Smale model, where unconditional flocking is guaranteed if the influence function decays sufficiently slowly at infinity. We shall prove the following sufficient condition for the validity of (15).

Corollary 1. Let the influence function $\psi=\psi(s)$ be such that

$$
\liminf _{s \rightarrow \infty} \frac{\psi(s)}{s^{\alpha}}>0
$$

for some $\alpha>-1$. Then all solutions of (5) exhibit asymptotic flocking as defined by (13), regardless of the particular values of $\bar{\tau}, R_{v}^{0}, d_{x}^{0}$ and $d_{v}^{0}$.

The above condition is almost equivalent to the "classical" unconditional flocking condition $\int^{+\infty} \psi(s) \mathrm{d} s=$ $+\infty$ for the Cucker-Smale model and the Motsch-Tadmor model [19] which corresponds to (5) with $\tau \equiv 0$. Indeed, the nonintegrability of $\psi$ at infinity is implied by (17) with any $\alpha \geq-1$, so that Corollary 1 only excludes influence functions that decay like $s^{-1}$. From this point of view, our result is very close to optimal. In particular, for the influence function

$$
\psi(s)=\frac{1}{\left(1+s^{2}\right)^{\beta}} \quad \text { for } s \geq 0,
$$

which was considered in the original works $[5,6]$, Corollary 1 guarantees unconditional flocking whenever $\beta<1 / 2$. This is precisely the setting that leads to unconditional flocking in the original Cucker-Smale model, and with $\beta>1 / 2$ asymptotic flocking may or may not take place, depending on the initial datum.

The last part of the paper is devoted to the proof of asymptotic consensus and flocking in the mean-field limits of the Hegselmann-Krause model (1) and the Cucker-Smale system (5). Letting $N \rightarrow \infty$ in (1) leads to the conservation law

$$
\partial_{t} f+\nabla_{x} \cdot(F[f] f)=0
$$

for the time-dependent probability measure $f=f(t, x)$ which describes the probability of finding an agent at time $t \geq 0$ located at $x \in \mathbb{R}^{d}$. The operator $F=F[f]$ is defined as

$$
F[f](t, x):=\frac{\int_{\mathbb{R}^{d}} \psi(|x-y|)(y-x) f(t-\tau(t), y) \mathrm{d} y}{\int_{\mathbb{R}^{d}} \psi(|x-y|) f(t-\tau(t), y) \mathrm{d} y} .
$$

The system (19)-(20) is equipped with the initial datum $f(t)=f^{0}(t), t \in[-\bar{\tau}, 0]$, with $f^{0} \in C\left([-\bar{\tau}, 0], \mathbb{P}\left(\mathbb{R}^{d}\right)\right)$, where $\mathbb{P}\left(\mathbb{R}^{d}\right)$ denotes the set of probability measures on $\mathbb{R}^{d}$. We shall assume that the initial datum is uniformly compactly supported, i.e.,

$$
\sup _{s \in[-\bar{\tau}, 0]} d_{x}\left[f^{0}(s)\right]<\infty
$$

where the diameter $d_{x}[h]$ for a probability measure $h \in \mathbb{P}\left(\mathbb{R}^{d}\right)$ is defined as

$$
d_{x}[h]:=\sup \{|x-y|, x, y \in \operatorname{supp} h\} .
$$

In Section 5 we shall prove the following theorem, which is a direct consequence of a stability estimate in terms of the Monge-Kantorowich-Rubinstein distance, combined with the fact that the consensus estimates derived in Section 3 are uniform with respect to the number of agents $N \in \mathbb{N}$.

Theorem 3. Let the assumptions (9) on $\psi=\psi(s)$ and (10) on $\tau=\tau(t)$ be verified. Then all solutions $f=f(t)$ of (19) with compactly supported initial datum (21) reach global asymptotic consensus in the sense

$$
\lim _{t \rightarrow \infty} d_{x}[f(t)]=0
$$

and the decay is exponential. 
The mean-field limit of the Cucker-Smale system (5) is given, in its strong formulation, by the kinetic equation

$$
\partial_{t} g+v \cdot \nabla_{x} g+\nabla_{v} \cdot(G[g] g)=0
$$

with

$$
G[g](t, x):=\frac{\int_{\mathbb{R}^{d}} \int_{\mathbb{R}^{d}} \psi(|x-y|)(w-v) f(t-\tau(t), y, w) \mathrm{d} y \mathrm{~d} w}{\int_{\mathbb{R}^{d}} \int_{\mathbb{R}^{d}} \psi(|x-y|) f(t-\tau(t), y, w) \mathrm{d} y \mathrm{~d} w} .
$$

Here the time-dependent probability measure $g=g(t, x, v)$ describes the probability of finding an agent at time $t \geq 0$ located at $x \in \mathbb{R}^{d}$ with velocity $v \in \mathbb{R}^{d}$. The initial datum $g(t)=g^{0}(t), t \in[-\bar{\tau}, 0]$, with $g^{0} \in C\left([-\tau, 0], \mathbb{P}\left(\mathbb{R}^{d} \times \mathbb{R}^{d}\right)\right)$, is again assumed to be uniformly compactly supported in the sense that there exists a compact set $K \subset \mathbb{R}^{d} \times \mathbb{R}^{d}$ such that

$$
\operatorname{supp} g^{0}(t) \subset K \quad \text { for all } t \in[-\bar{\tau}, 0] \text {. }
$$

With a slight abuse of notation, define the position and velocity diameters of $\operatorname{supp} g(t)$ as

$$
d_{x}[g(t)]:=\sup \left\{|x-y| ; x, y \in \operatorname{supp}_{x} g(t)\right\}, \quad d_{v}[g(t)]:=\sup \left\{|v-w| ; v, w \in \operatorname{supp}_{v} g(t)\right\},
$$

where $\operatorname{supp}_{x} g$ and, resp., $\operatorname{supp}_{v} g$, are projections of supp $g$ onto the $x$ - and, resp., $v$-variables. The velocity radius is defined as

$$
R_{v}[g(t)]:=\sup \left\{|w|, w \in \operatorname{supp}_{v} g(t)\right\} .
$$

We then have the following flocking result.

Theorem 4. Let the assumptions (9) on $\psi=\psi(s)$ and (10) on $\tau=\tau(t)$ be verified. Moreover, assume that there exists $C \in(0,1)$ such that

$$
1-C=\left(1-\Psi\left(\bar{\tau} R_{v}^{0}+d_{x}^{0}+\frac{d_{v}^{0}}{C}\right)\right) e^{C \bar{\tau}}
$$

with $\Psi$ defined in (14) and

$$
R_{v}^{0}:=\max _{s \in[-\bar{\tau}, 0]} R_{v}\left[g^{0}(s)\right], \quad d_{x}^{0}:=\max _{s \in[-\bar{\tau}, 0]} d_{x}\left[g^{0}(s)\right], \quad d_{v}^{0}:=\max _{s \in[-\bar{\tau}, 0]} d_{v}\left[g^{0}(s)\right] .
$$

Then the solution of (22) subject to the compactly supported initial datum (24) exhibits asymptotic flocking in the sense

$$
\lim _{t \rightarrow \infty} d_{v}[g(t)]=0, \quad \sup _{t \geq 0} d_{x}[g(t)]<\infty .
$$

Moreover, the decay of the velocity diameter to zero as $t \rightarrow \infty$ is exponential with rate $C$.

Again, note that assumption (25) is satisfied regardless of the values of $\bar{\tau}, R_{v}^{0}, d_{x}^{0}$ and $d_{v}^{0}$ whenever the influence function satisfies the condition (17).

\section{Asymptotic consensus for the Hegselmann-Krause model}

We define the radius of the group $R_{x}=R_{x}(t)$ by

$$
R_{x}(t):=\max _{1 \leq i \leq N}\left|x_{i}(t)\right| .
$$

The following lemma shows that the radius is bounded uniformly in time by the radius of the initial datum, defined as

$$
R_{x}^{0}:=\max _{t \in[-\bar{\tau}, 0]} R_{x}(t)
$$


Lemma 1. Let the initial datum $x^{0} \in C\left([-\bar{\tau}, 0] ; \mathbb{R}^{d}\right)^{N}$ and let $R_{x}^{0}$ be given by (28). Then, along the solutions of (1)-(3), the diameter $R_{x}=R_{x}(t)$ defined in (27) satisfies

$$
R_{x}(t) \leq R_{x}^{0} \quad \text { for all } t \geq 0 .
$$

The proof of a slight generalization of [2, Lemma 2.2] for the case of variable time delay. We give it here for the sake of the reader.

Proof. Let us fix some $\varepsilon>0$. We shall prove that for all $t \geq 0$

$$
R_{x}(t)<R_{x}^{0}+\varepsilon .
$$

Obviously, $R_{x}(0) \leq R_{x}^{0}$, so that by continuity, (29) holds on the maximal interval $[0, T)$ for some $T>0$. For contradiction, let us assume that $T<+\infty$. Then we have

$$
\lim _{t \rightarrow T-} R_{x}(t)=R_{x}^{0}+\varepsilon
$$

However, for any $i=1, \ldots, N$, we have

$$
\begin{aligned}
\frac{1}{2} \frac{\mathrm{d}}{\mathrm{d} t}\left|x_{i}(t)\right|^{2} & =\sum_{j \neq i} \psi_{i j}(t)\left[x_{j}(t-\tau(t))-x_{i}(t)\right] \cdot x_{i}(t) \\
& =\sum_{j \neq i} \psi_{i j}(t)\left[x_{j}(t-\tau(t)) \cdot x_{i}(t)-\left|x_{i}(t)\right|^{2}\right] .
\end{aligned}
$$

By definition, we have $\left|x_{j}(t-\tau(t))\right|<R_{x}^{0}+\varepsilon$ for all $j \in\{1, \ldots, N\}$ and $t<T$, so that with (4) and an application of the Cauchy-Schwarz inequality we arrive at

$$
\frac{1}{2} \frac{\mathrm{d}}{\mathrm{d} t}\left|x_{i}(t)\right|^{2} \leq\left(R_{x}^{0}+\varepsilon\right)\left|x_{i}(t)\right|-\left|x_{i}(t)\right|^{2} .
$$

Now, if $\left|x_{i}(t)\right| \neq 0$, we use the identity $\frac{1}{2} \frac{\mathrm{d}\left|x_{i}(t)\right|^{2}}{\mathrm{~d} t}=\left|x_{i}(t)\right| \frac{\mathrm{d}\left|x_{i}(t)\right|}{\mathrm{d} t}$ and divide the above inequality by $\left|x_{i}(t)\right|$. On the other hand, if $\left|x_{i}(t)\right| \equiv 0$ on an open subinterval of $(0, T)$, then $\frac{\mathrm{d}\left|x_{i}(t)\right|}{\mathrm{d} t} \equiv 0 \leq R_{x}^{0}+\varepsilon-\left|x_{i}(t)\right|$ on this subinterval. Thus, we obtain

$$
\frac{\mathrm{d}}{\mathrm{d} t}\left|x_{i}(t)\right| \leq R_{x}^{0}+\varepsilon-\left|x_{i}(t)\right| \quad \text { a.e. on }(0, T)
$$

which implies

$$
\left|x_{i}(t)\right| \leq\left(\left|x_{i}(0)\right|-\left(R_{x}^{0}+\varepsilon\right)\right) e^{-t}+R_{x}^{0}+\varepsilon \quad \text { for } t<T .
$$

Consequently, with $\left|x_{i}(0)\right| \leq R_{x}^{0}$,

$$
\lim _{t \rightarrow T-} \max _{1 \leq i \leq N}\left|x_{i}(t)\right| \leq-\varepsilon e^{-T}+R_{x}^{0}+\varepsilon<R_{x}^{0}+\varepsilon
$$

which is a contradiction to (30). We conclude that, indeed, $T=\infty$, and complete the proof by taking the limit $\varepsilon \rightarrow 0$.

The following geometric result is based on the convexity property (4) of the normalized communication weights (2).

Lemma 2. Let $N \geq 3$ and $\left\{x_{1}, \ldots, x_{N}\right\} \subset \mathbb{R}^{d}$ be any set of vectors in $\mathbb{R}^{d}$ and denote $d_{x}$ its diameter,

$$
d_{x}:=\max _{1 \leq i, j \leq N}\left|x_{i}-x_{j}\right| .
$$


Fix $i, k \in\{1,2, \cdots, N\}$ such that $i \neq k$ and let $\eta_{j}^{i} \geq 0$ for all $j \in\{1,2, \cdots, N\} \backslash\{i\}$, and $\eta_{j}^{k} \geq 0$ for all $j \in\{1,2, \cdots, N\} \backslash\{k\}$, such that

$$
\sum_{j \neq i} \eta_{j}^{i}=1, \quad \sum_{j \neq k} \eta_{j}^{k}=1
$$

Let $\mu \geq 0$ be such that

$$
0 \leq \mu \leq \min \left\{\min _{j \neq i} \eta_{j}^{i}, \min _{j \neq k} \eta_{j}^{k}\right\}
$$

Then

$$
\left|\sum_{j \neq i} \eta_{j}^{i} x_{j}-\sum_{j \neq k} \eta_{j}^{k} x_{j}\right| \leq(1-(N-2) \mu) d_{x} .
$$

Proof. For $j, \ell \in\{1,2, \cdots, N\} \backslash\{i\}$ we set $\xi_{j \ell}^{i}:=\mu$ if $\ell \neq j$ and $\xi_{j j}^{i}:=1-(N-2) \mu$. Moreover, define

$$
\bar{x}_{j}^{i}:=\sum_{\ell \neq i} \xi_{j \ell}^{i} x_{\ell} \quad \text { for } j \in\{1,2, \cdots, N\} \backslash\{i\} .
$$

Then the vector $\sum_{j \neq i} \eta_{j}^{i} x_{j}$ can be written as the convex combination of the vectors $\bar{x}_{j}^{i}$,

$$
\sum_{j \neq i} \eta_{j}^{i} x_{j}=\sum_{j \neq i} \lambda_{j}^{i} \bar{x}_{j}^{i}
$$

with the coefficients

$$
\lambda_{j}^{i}=\frac{\eta_{j}^{i}-\mu}{1-(N-1) \mu} \geq 0, \quad \sum_{j \neq i} \lambda_{j}^{i}=1 .
$$

Similarly, for $j, \ell \in\{1,2, \cdots, N\} \backslash\{k\}$ we set $\xi_{j \ell}^{k}:=\mu$ if $\ell \neq j$ and $\xi_{j j}^{k}:=1-(N-2) \mu$. Define

$$
\bar{x}_{j}^{k}:=\sum_{\ell \neq k} \xi_{j \ell}^{k} x_{\ell} \quad \text { for } j \in\{1,2, \cdots, N\} \backslash\{k\},
$$

and again observe that the vector $\sum_{j \neq k} \eta_{j}^{k} x_{j}$ can be written as the convex combination of the vectors $\bar{x}_{j}^{k}$,

$$
\sum_{j \neq k} \eta_{j}^{k} x_{j}=\sum_{j \neq k} \lambda_{j}^{k} \bar{x}_{j}^{k}
$$

with the coefficients

$$
\lambda_{j}^{k}=\frac{\eta_{j}^{k}-\mu}{1-(N-1) \mu} \geq 0, \quad \sum_{j \neq k} \lambda_{j}^{k}=1 .
$$

Obviously, due to (33) and (35), the estimate (31) is verified as soon as we prove

$$
\left|\bar{x}_{a}^{i}-\bar{x}_{b}^{k}\right| \leq(1-(N-2) \mu) d_{x}
$$

for all $a \in\{1,2, \cdots, N\} \backslash\{i\}$ and $b \in\{1,2, \cdots, N\} \backslash\{k\}$. With (32) and (34) we have

$$
\begin{aligned}
\bar{x}_{a}^{i}-\bar{x}_{b}^{k} & =\sum_{\ell \neq i} \xi_{a \ell}^{i} x_{\ell}-\sum_{\ell \neq k} \xi_{b \ell}^{k} x_{\ell} \\
& =\mu \sum_{\ell \neq i, a} x_{\ell}+(1-(N-2) \mu) x_{a}-\mu \sum_{\ell \neq k, b} x_{\ell}-(1-(N-2) \mu) x_{b} \\
& =\mu\left(x_{k}-x_{i}\right)+(1-(N-1) \mu)\left(x_{a}-x_{b}\right) .
\end{aligned}
$$


With the triangle inequality we then readily obtain

$$
\left|\bar{x}_{a}^{i}-\bar{x}_{b}^{k}\right| \leq(1-(N-2) \mu) d_{x} .
$$

The following Gronwall-Halanay type inequality is a generalization of [2, Lemma 2.5] for variable time delay.

Lemma 3. Let $\tau=\tau(t)$ satisfy the assumptions (10). Let $u \in C([-\bar{\tau}, \infty))$ be a nonnegative continuous function with piecewise continuous derivative on $(0, \infty)$, such that for some constant $a \in(0,1)$ the differential inequality is satisfied,

$$
\frac{\mathrm{d}}{\mathrm{d} t} u(t) \leq(1-a) u(t-\tau(t))-u(t) \quad \text { for almost all } t>0 .
$$

Then there exists a unique solution $C \in(0, a)$ of the equation

$$
1-C=(1-a) e^{C \bar{\tau}}
$$

and the estimate holds

$$
u(t) \leq\left(\max _{s \in[-\bar{\tau}, 0]} u(s)\right) e^{-C t} \quad \text { for all } t \geq 0 .
$$

Proof. We denote

$$
\bar{u}:=\max _{s \in[-\bar{\tau}, 0]} u(s), \quad w(t):=\bar{u} e^{-C t},
$$

and for any fixed $\lambda>1$ set

$$
\mathscr{S}_{\lambda}:=\{t \geq 0: u(s) \leq \lambda w(s) \text { for } \quad s \in[0, t)\} .
$$

Since $0 \in \mathscr{S}_{\lambda}, T_{\lambda}:=\sup \mathscr{S}_{\lambda} \geq 0$ exists. We claim that

$$
T_{\lambda}=\infty \quad \text { for any } \lambda>1 .
$$

For contradiction, assume $T_{\lambda}<\infty$ for some $\lambda>1$. Then clearly $\tau\left(T_{\lambda}\right)>0$, since otherwise we would have

$$
\frac{\mathrm{d}}{\mathrm{d} t+} u(t) \leq \lim _{t \rightarrow T_{\lambda}+}(1-a) u(t-\tau(t))-u(t)=-a u\left(T_{\lambda}\right)=-a \lambda w\left(T_{\lambda}\right)<0,
$$

which contradicts the definition of $T_{\lambda}$. Therefore, due to the continuity of $u=u(t)$ and $\tau=\tau(t)$, there exists some $T_{\lambda}^{*}>T_{\lambda}$ such that $u$ is differentiable at $T_{\lambda}^{*}$ and

$$
u\left(T_{\lambda}^{*}\right)>\lambda w\left(T_{\lambda}^{*}\right), \quad \frac{\mathrm{d}}{\mathrm{d} t} u\left(T_{\lambda}^{*}\right)>\lambda \frac{\mathrm{d}}{\mathrm{d} t} w\left(T_{\lambda}^{*}\right), \quad \tau\left(T_{\lambda}^{*}\right)>T_{\lambda}^{*}-T_{\lambda} .
$$

Note that $w$ satisfies

$$
w(t-\tau(t))=e^{C \tau(t)} w(t) \quad \text { and } \quad \frac{\mathrm{d}}{\mathrm{d} t} w(t)=-C w(t),
$$

for all $t>0$. Moreover, it follows from (36) that

$$
\frac{\mathrm{d}}{\mathrm{d} t} u\left(T_{\lambda}^{*}\right) \leq(1-a) u\left(T_{\lambda}^{*}-\tau\left(T_{\lambda}^{*}\right)\right)-u\left(T_{\lambda}^{*}\right) .
$$

We now consider the following two cases: 
- If $T_{\lambda}^{*}-\tau\left(T_{\lambda}^{*}\right) \leq 0$, then by (39) we have $u\left(T_{\lambda}^{*}-\tau\left(T_{\lambda}^{*}\right)\right) \leq \bar{u}$ and $u\left(T_{\lambda}^{*}\right)>\lambda w\left(T_{\lambda}^{*}\right)$ by (40), so that we estimate the right-hand side of (42) by

$$
\begin{aligned}
\frac{\mathrm{d}}{\mathrm{d} t} u\left(T_{\lambda}^{*}\right) & \leq(1-a) \bar{u}-u\left(T_{\lambda}^{*}\right) \\
& <(1-a) \lambda w(0)-\lambda w\left(T_{\lambda}^{*}\right) \\
& \leq\left((1-a) e^{C \bar{\tau}}-1\right) \lambda w\left(T_{\lambda}^{*}\right) .
\end{aligned}
$$

For the third line we used the inequality $w(0) \leq w\left(T_{\lambda}^{*}\right) e^{C \bar{\tau}}$ implied by $T_{\lambda}^{*} \leq \tau\left(T_{\lambda}^{*}\right) \leq \bar{\tau}$. With the identities (37) and (41), we obtain

$$
\frac{\mathrm{d}}{\mathrm{d} t} u\left(T_{\lambda}^{*}\right)<-C \lambda w\left(T_{\lambda}^{*}\right)=\lambda \frac{\mathrm{d}}{\mathrm{d} t} w\left(T_{\lambda}^{*}\right),
$$

which is a contradiction to $(40)$.

- If $T_{\lambda}^{*}-\tau\left(T_{\lambda}^{*}\right)>0$, we have $u\left(T_{\lambda}^{*}-\tau\left(T_{\lambda}^{*}\right)\right) \leq \lambda w\left(T_{\lambda}^{*}-\tau\left(T_{\lambda}^{*}\right)\right)$ since, due to $(40), T_{\lambda}^{*}-\tau\left(T_{\lambda}^{*}\right)<T_{\lambda}$. Then (42) gives

$$
\begin{aligned}
\frac{\mathrm{d}}{\mathrm{d} t} u\left(T_{\lambda}^{*}\right) & \leq(1-a) \lambda w\left(T_{\lambda}^{*}-\tau\left(T_{\lambda}^{*}\right)\right)-\lambda w\left(T_{\lambda}^{*}\right) \\
& =\left((1-a) e^{C \tau\left(T_{\lambda}^{*}\right)}-1\right) \lambda w\left(T_{\lambda}^{*}\right) \\
& \leq\left((1-a) e^{C \bar{\tau}}-1\right) \lambda w\left(T_{\lambda}^{*}\right) .
\end{aligned}
$$

Then, using the same argument as in the previous case, we obtain a contradiction to (40).

We conclude that, for every $\lambda>1, T_{\lambda}=\infty$ and $u(t) \leq \lambda w(t)$ for all $t \geq 0$. Passing to the limit $\lambda \rightarrow 1$ yields the claim (38).

We are now prepared to prove Theorem 1.

Proof. For the sake of legibility, let us introduce the shorthand notation $\widetilde{x}_{j}:=x_{j}(t-\tau(t))$, while $x_{j}$ means $x_{j}(t)$.

The uniform bound on the radius $R_{x}=R_{x}(t)$ of the solution provided by Lemma 1 gives for all $i, j \in$ $\{1,2, \cdots, N\}$

$$
\left|\widetilde{x}_{j}-x_{i}\right| \leq\left|\widetilde{x}_{j}\right|+\left|\widetilde{x}_{i}\right| \leq 2 R_{x}^{0} \quad \text { for all } t \geq 0 .
$$

Consequently, defining

$$
\underline{\psi}:=\min _{s \in\left[0,2 R_{x}^{0}\right]} \psi(s),
$$

we have $\psi\left(\left|\widetilde{x}_{j}-x_{i}\right|\right) \geq \underline{\psi}$, and

$$
\psi_{i j}=\frac{\psi\left(\left|\widetilde{x}_{j}-x_{i}\right|\right)}{\sum_{\ell \neq i} \psi\left(\left|\widetilde{x}_{\ell}-x_{i}\right|\right)} \geq \frac{\underline{\psi}}{N-1} .
$$

Note that due to the assumption (9), we have $\underline{\psi}>0$.

Due to the continuity of the trajectories $x_{i}=x_{i}(t)$, there is an at most countable system of open, mutually disjoint intervals $\left\{\mathscr{I}_{\sigma}\right\}_{\sigma \in \mathbb{N}}$ such that

$$
\bigcup_{\sigma \in \mathbb{N}} \overline{\mathscr{I}_{\sigma}}=[0, \infty)
$$

and for each $\sigma \in \mathbb{N}$ there exist indices $i(\sigma), k(\sigma)$ such that

$$
d_{x}(t)=\left|x_{i(\sigma)}(t)-x_{k(\sigma)}(t)\right| \quad \text { for } t \in \mathscr{I}_{\sigma} .
$$


Then, using the abbreviated notation $i:=i(\sigma), k:=k(\sigma)$, we have for every $t \in \mathscr{I}_{\sigma}$,

$$
\begin{aligned}
\frac{1}{2} \frac{\mathrm{d}}{\mathrm{d} t} d_{x}(t)^{2} & =\left(\dot{x}_{i}-\dot{x}_{k}\right) \cdot\left(x_{i}-x_{k}\right) \\
& =\left(\sum_{j \neq i} \psi_{i j}\left(\widetilde{x}_{j}-x_{i}\right)-\sum_{j \neq k} \psi_{k j}\left(\widetilde{x}_{j}-x_{k}\right)\right) \cdot\left(x_{i}-x_{k}\right) \\
& =\left(\sum_{j \neq i} \psi_{i j} \widetilde{x}_{j}-\sum_{j \neq k} \psi_{k j} \widetilde{x}_{j}\right) \cdot\left(x_{i}-x_{k}\right)-\left|x_{i}-x_{k}\right|^{2},
\end{aligned}
$$

where we used the convexity property of the normalized weights (4). We now use (44) and Lemma 2 with $\mu:=\frac{\psi}{N-1}$, which gives

$$
\left|\sum_{j \neq i} \psi_{i j} \widetilde{x}_{j}-\sum_{j \neq k} \psi_{k j} \widetilde{x}_{j}\right| \leq(1-(N-2) \mu) d_{x}(t-\tau(t)) .
$$

Consequently, with the Cauchy-Schwartz inequality we have

$$
\frac{1}{2} \frac{\mathrm{d}}{\mathrm{d} t} d_{x}(t)^{2} \leq(1-(N-2) \mu) d_{x}(t-\tau) d_{x}(t)-d_{x}(t)^{2}
$$

which implies that for almost all $t>0$,

$$
\frac{\mathrm{d}}{\mathrm{d} t} d_{x}(t) \leq(1-(N-2) \mu) d_{x}(t-\tau(t))-d_{x}(t)
$$

An application of Lemma 3 with $a:=(N-2) \mu=\frac{N-2}{N-1} \underline{\psi} \in(0,1)$ gives then the exponential decay

$$
d_{x}(t) \leq\left(\max _{s \in[-\bar{\tau}, 0]} d_{x}(s)\right) e^{-C t} \quad \text { for } t \geq 0
$$

where $C$ is the unique solution of (37). We note that $a$ increases with increasing $N$ (if $\psi$ is held constant), and so does $C$. Consequently, the exponential decay rate $C$ improves with increasing $N$.

\section{Asymptotic flocking for the Cucker-Smale model}

The method develop in Section 3 can be easily extended for the Cucker-Smale model (5), as we demonstrate in the proof of Theorem 2 below.

Proof. First, note that the proof of Lemma 1 applies mutatis mutandis for the velocity variable in (5), providing the uniform bound

$$
R_{v}(t):=\max _{1 \leq i \leq N}\left|v_{i}(t)\right| \leq R_{v}^{0} \quad \text { for all } t \geq 0,
$$

with $R_{v}^{0}:=\max _{t \in[-\bar{\tau}, 0]} R_{v}(t)$.

Let $C \in(0,1)$ be given by (15). With $d_{v}^{0}>0$ given by (26) and due to the continuity of $d_{v}=d_{v}(t)$, there exists some $T>0$ such that

$$
\int_{0}^{t} d_{v}(s) \mathrm{d} s<\frac{d_{v}^{0}}{C} \quad \text { for all } t<T
$$


We claim that $T=\infty$. For contradiction, assume that (46) holds only until some finite $T>0$. Then we have

$$
\int_{0}^{T} d_{v}(s) \mathrm{d} s=\frac{d_{v}^{0}}{C}
$$

By the first equation of (5) we readily have

$$
d_{x}(t) \leq d_{x}^{0}+\int_{0}^{t} d_{v}(s) \mathrm{d} s
$$

so that (46) implies for all $t<T$,

$$
d_{x}(t) \leq d_{x}^{0}+\int_{0}^{t} d_{v}(s) \mathrm{d} s<d_{x}^{0}+\frac{d_{v}^{0}}{C}
$$

Moreover, using the estimate

$$
\left|\widetilde{x}_{j}-x_{j}\right|=\left|\int_{t-\tau(t)}^{t} \dot{x}_{j}(s) \mathrm{d} s\right| \leq \int_{t-\tau(t)}^{t}\left|v_{j}(s)\right| \mathrm{d} s \leq \bar{\tau} R_{v}^{0},
$$

provided by (45), we have for any $i, j \in\{1,2, \cdots, N\}, i \neq j$,

$$
\left|\widetilde{x}_{j}-x_{i}\right| \leq\left|\widetilde{x}_{j}-x_{j}\right|+\left|x_{j}-x_{i}\right| \leq \bar{\tau} R_{v}^{0}+d_{x}(t) \leq \bar{\tau} R_{v}^{0}+d_{x}^{0}+\frac{d_{v}^{0}}{C} .
$$

Then by the definition (14) of $\Psi$,

$$
\psi\left(\left|\widetilde{x}_{j}-x_{i}\right|\right) \geq \Psi\left(\bar{\tau} R_{v}^{0}+d_{x}^{0}+\frac{d_{v}^{0}}{C}\right),
$$

and by the universal bound $\psi \leq 1$,

$$
\psi_{i j}=\frac{\psi\left(\left|\widetilde{x}_{j}-x_{i}\right|\right)}{\sum_{\ell \neq i} \psi\left(\left|\widetilde{x}_{\ell}-x_{i}\right|\right)} \geq \frac{1}{N-1} \Psi\left(\bar{\tau} R_{v}^{0}+d_{x}^{0}+\frac{d_{v}^{0}}{C}\right) .
$$

Similarly as in the proof of Theorem 1, we have for $t<T$ such that $d_{v}=\left|v_{i}-v_{k}\right|$ on some neighborhood of $t$,

$$
\begin{aligned}
\frac{1}{2} \frac{\mathrm{d}}{\mathrm{d} t} d_{v}(t)^{2} & =\left(\sum_{j \neq i} \psi_{i j} \widetilde{v}_{j}-\sum_{j \neq k} \psi_{k j} \widetilde{v}_{j}\right) \cdot\left(v_{i}-v_{k}\right)-\left|v_{i}-v_{k}\right|^{2} \\
& \leq\left|\sum_{j \neq i} \psi_{i j} \widetilde{v}_{j}-\sum_{j \neq k} \psi_{k j} \widetilde{v}_{j}\right| d_{v}(t)-d_{v}(t)^{2}
\end{aligned}
$$

We now use (48) and Lemma 2 with $\mu:=\frac{1}{N-1} \Psi\left(\bar{\tau} R_{v}^{0}+d_{x}^{0}+\frac{d_{v}^{0}}{C}\right)$, which gives

$$
\left|\sum_{j \neq i} \psi_{i j} \widetilde{v}_{j}-\sum_{j \neq k} \psi_{k j} \widetilde{v}_{j}\right| \leq(1-(N-2) \mu) d_{v}(t-\tau(t)) .
$$

Consequently, (49) implies that for almost all $t>0$,

$$
\frac{\mathrm{d}}{\mathrm{d} t} d_{v}(t) \leq(1-(N-2) \mu) d_{v}(t-\tau(t))-d_{v}(t) .
$$


An application of Lemma 3 with

$$
a:=(N-2) \mu=\frac{N-2}{N-1} \psi\left(\bar{\tau} R_{v}^{0}+d_{x}^{0}+\frac{d_{v}^{0}}{C}\right),
$$

recalling (15), gives

$$
d_{v}(t) \leq d_{v}^{0} e^{-C t} \quad \text { for all } t<T
$$

But then

$$
\int_{0}^{T} d_{v}(s) \mathrm{d} s \leq d_{v}^{0} \int_{0}^{T} e^{-C s} \mathrm{~d} s=\frac{d_{v}^{0}}{C}\left(1-e^{-C T}\right)<\frac{d_{v}^{0}}{C},
$$

which is a contradiction to (47). Thus, we conclude that $T=\infty$, i.e., that (46) holds for all $t>0$. Then also (50) holds for all $t>0$, and, moreover, $d_{x}=d_{x}(t)$ is uniformly bounded by $d_{x}^{0}+\frac{d_{v}^{0}}{C}$.

Finally, we provide the proof of Corollary 1. Clearly, (15) is solvable for $C \in(0,1)$ whenever $\inf _{s \in(0, \infty)} \psi(s)>$ 0 . Therefore, let us consider $\psi=\psi(s)$ such that $\Psi(r)=\inf _{s \in(0, r)} \psi(s) \rightarrow 0$ as $r \rightarrow \infty$. Then we have

$$
\lim _{C \rightarrow 0+}\left(1-\frac{N-2}{N-1} \Psi\left(\bar{\tau} R_{v}^{0}+d_{x}^{0}+\frac{d_{v}^{0}}{C}\right)\right) e^{C \bar{\tau}}=1,
$$

for any $d_{v}^{0}>0$ and nonnegative $R_{v}^{0}, d_{x}^{0}$ and $\bar{\tau}$. On the other hand, since by assumption $\psi(s)>0$ for all $s \geq 0$, the above expression is strictly positive for $C=1$. Assumption (17) implies that $\Psi(r) \geq c r^{\alpha}$ for some $c>0$ and all $r>0$ large enough. Denoting $\Phi(r):=c r^{\alpha}$, we have for $\alpha \in(0,1)$,

$$
\frac{\mathrm{d}}{\mathrm{d} C}\left[\left(1-\frac{N-2}{N-1} \Phi\left(\bar{\tau} R_{v}^{0}+d_{x}^{0}+\frac{d_{v}^{0}}{C}\right)\right) e^{C \bar{\tau}}\right]_{C=0+}=-\infty,
$$

again for any $d_{v}^{0}>0$ and nonnegative $R_{v}^{0}, d_{x}^{0}$ and $\bar{\tau}$. Consequently, using (17) we have

$$
1-C>\left(1-\frac{N-2}{N-1} \Phi\left(\bar{\tau} R_{v}^{0}+d_{x}^{0}+\frac{d_{v}^{0}}{C}\right)\right) e^{C \bar{\tau}} \geq\left(1-\frac{N-2}{N-1} \Psi\left(\bar{\tau} R_{v}^{0}+d_{x}^{0}+\frac{d_{v}^{0}}{C}\right)\right) e^{C \bar{\tau}}
$$

for all $C>0$ small enough, and we conclude that there exists some $C \in(0,1)$ such that (15) holds.

\section{Consensus and flocking in the mean-field limit}

Our results for the mean-field limit systems (19)-(20) and (22)-(23) are based on the well-posedness theory in measures developed in [2, Section 3]. In particular, existence and uniqueness of measure-valued solutions for the Cucker-Smale system (22)-(23) was proved there, together with continuous dependence on the initial datum. The proof uses the framework developed in [1] and is based on local Lipschitz continuity of the operator $F=F[f]$ given by (20). Without going into details, we note that the proof can be easily adapted to provide analogous results for the Hegselmann-Krause system (19)-(20). Instead, we merely restate the stability results in Wasserstein distance for the two systems, which are essential for our proof of asymptotic consensus and flocking. For their proof we refer to [2, Theorem 3.6].

Theorem 5. Let $f_{1}, f_{2} \in C\left([0, T] ; \mathbb{P}\left(\mathbb{R}^{d}\right)\right)$ be two measure-valued solutions of (19)-(20) on the time interval $[0, T]$, subject to the compactly supported initial data $f_{1}^{0}, f_{2}^{0} \in C\left([-\bar{\tau}, 0] ; \mathbb{P}_{1}\left(\mathbb{R}^{d}\right)\right)$. Then there exists a constant $L=L(T)$ such that

$$
\mathscr{W}_{1}\left(f_{1}(t), f_{2}(t)\right) \leq L \max _{s \in[-\bar{\tau}, 0]} \mathscr{W}_{1}\left(f_{1}^{0}(s), f_{2}^{0}(s)\right) \quad \text { for } \quad t \in[0, T]
$$

where $\mathscr{W}_{1}\left(f_{1}(t), f_{2}(t)\right)$ denotes the 1-Wasserstein (or Monge-Kantorovich-Rubinstein) distance [26] of the probability measures $f_{1}(t), f_{2}(t)$. 
Theorem 6. Let $g_{1}, g_{2} \in C\left([0, T] ; \mathbb{P}\left(\mathbb{R}^{d} \times \mathbb{R}^{d}\right)\right)$ be two measure-valued solutions of (22)-(23) on the time interval $[0, T]$, subject to the compactly supported initial data $g_{1}^{0}, g_{2}^{0} \in C\left([-\bar{\tau}, 0] ; \mathbb{P}_{1}\left(\mathbb{R}^{d} \times \mathbb{R}^{d}\right)\right)$. Then there exists a constant $L=L(T)$ such that

$$
\mathscr{W}_{1}\left(g_{1}(t), g_{2}(t)\right) \leq L \max _{s \in[-\bar{\tau}, 0]} \mathscr{W}_{1}\left(g_{1}^{0}(s), g_{2}^{0}(s)\right) \quad \text { for } \quad t \in[0, T] .
$$

We are now in position to provide a proof of Theorem 3.

Proof. Fixing an initial datum $f^{0} \in C\left([-\bar{\tau}, 0], \mathbb{P}\left(\mathbb{R}^{d}\right)\right)$, uniformly compactly supported in the sense of (21), we construct $\left\{f_{N}^{0}\right\}_{N \in \mathbb{N}}$ a family of $N$-particle approximations of $f^{0}$, i.e.,

$$
f_{N}^{0}(s, x):=\frac{1}{N} \sum_{i=1}^{N} \delta\left(x-x_{i}^{0}(s)\right) \quad \text { for } s \in[-\bar{\tau}, 0],
$$

where the $x_{i}^{0} \in C\left([-\bar{\tau}, 0] ; \mathbb{R}^{d}\right)$ are chosen such that

$$
\max _{s \in[-\bar{\tau}, 0]} \mathscr{W}_{1}\left(f_{N}^{0}(s), f^{0}(s)\right) \rightarrow 0 \quad \text { as } \quad N \rightarrow \infty .
$$

Denoting then $x_{i}^{N}=x_{i}^{N}(t)$ the solution of the discrete Hegselmann-Krause system (1) subject to the initial datum $x_{i}^{0}=x_{i}^{0}(s), i=1, \ldots, N$, the proof of Theorem 1 gives exponential convergence to global consensus, i.e.,

$$
d_{x}(t) \leq\left(\max _{s \in[-\bar{\tau}, 0]} d_{x}(s)\right) e^{-C_{N} t} \quad \text { for } t \geq 0,
$$

with the diameter $d_{x}$ defined in (11), $C_{N}$ the unique solution of (37) with $a_{N}:=\frac{N-2}{N-1} \underline{\psi} \in(0,1)$ and $\underline{\psi}$ given by (43). Note that $C_{N}$ increases with $N$ and $\lim _{N \rightarrow \infty} C_{N}=C$, with $C$ the unique solution of (3) $\overline{7}$ ) with $a:=\underline{\psi}$. It is easy to check that the empirical measure

$$
f^{N}(t, x):=\frac{1}{N} \sum_{i=1}^{N} \delta\left(x-x_{i}^{N}(t)\right)
$$

is a measure valued solution of (1). For any fixed $T>0$, Theorem 5 provides the stability estimate

$$
\mathscr{W}_{1}\left(f(t), f^{N}(t)\right) \leq L \max _{s \in[-\bar{\tau}, 0]} \mathscr{W}_{1}\left(f^{0}(s), f_{N}^{0}(s)\right) \quad \text { for } \quad t \in[0, T),
$$

where the constant $L>0$ is independent of $N$. Thus, fixing $T>0$ and letting $N \rightarrow \infty$ implies $d_{x}[f(t)]=d_{x}(t)$ on $[0, T)$, and, consequently,

$$
d_{x}[f(t)] \leq\left(\max _{s \in[-\bar{\tau}, 0]} d_{x}\left[f^{0}(s)\right]\right) e^{-C t} \quad \text { for } t \in[0, T) .
$$

We conclude by noting that $T>0$ can be chosen arbitrarily and that the constant $C$ is independent of time. 6.

The proof of Theorem 4 is an obvious modification of the above proof, with stability provided by Theorem

\section{Acknowledgment}

JH acknowledges the support of the KAUST baseline funds. 


\section{References}

[1] J. Cañizo, J. Carrillo and J. Rosado, A well-posedness theory in measures for some kinetic models of collective motion, Math. Mod. Meth. Appl. Sci., 21 (2011), 515-539.

[2] Y.-P. Choi and J. Haskovec: Cucker-Smale model with normalized communication weights and time delay.. Kinetic and Related Models 10 (2017), 1011-1033.

[3] Y.-P. Choi and J. Haskovec: Hydrodynamic Cucker-Smale model with normalized communication weights and time delay.. SIAM J. Math. Anal., Vol. 51, No. 3 (2019), 2660-2685.

[4] Y.-P. Choi, A. Paolucci and C. Pignotti: Consensus of the Hegselmann-Krause opinion formation model with time delay. arXiv:1909.02795 (2019).

[5] F. Cucker and S. Smale, Emergent behaviour in flocks, IEEE T. on Automat. Contr., 52 (2007), $852-862$.

[6] F. Cucker and S. Smale, On the mathematics of emergence, Jap. J. Math., 2 (2007), 197-227.

[7] R. L. Dobrushin, Central limit theorem for non-stationary Markov chains. I, Theory Probab. Appl., 1:1 (1956), 65-80.

[8] R. Erban, J. Haskovec and Y. Sun, A Cucker-Smale model with noise and delay, SIAM J. Appl. Math., 76 (2016), 1535-1557.

[9] S.-Y. Ha and J.-G. Liu, A simple proof of the Cucker-Smale flocking dynamics and mean-field limit, Comm. Math. Sci., 7 (2009), 297-325.

[10] S.-Y. Ha and E. Tadmor, From particle to kinetic and hydrodynamic descriptions of flocking, Kinetic and Related models, 1 (2008), 315-335.

[11] H. Hamman: Swarm Robotics: A Formal Approach. Springer, 2018.

[12] J. Haskovec and I. Markou: Asymptotic flocking in the Cucker-Smale model with reaction-type delays in the non-oscillatory regime. Kin. Rel. Models 13(4), in press (2020).

[13] R. Hegselmann and U. Krause, Opinion dynamics and bounded confidence models, analysis, and simulation. J. Artif. Soc. Soc. Simul., 5, (2002), 1-24.

[14] R. Hegselmann and U. Krause: Consensus and Fragmentation of Opinions with a Focus on Bounded Confidence. The American Mathematical Monthly 126:8 (2019), 700-716.

[15] P.E. Jabin and S. Motsch: Clustering and asymptotic behavior in opinion formation. J. Differential Equations 257 (2014), 4165-4187.

[16] U. Krause: A discrete nonlinear and non-autonomous model of consensus formation. Communications in difference equations, 227-236 (2000).

[17] Y. Liu, and J. Wu, Flocking and asymptotic velocity of the Cucker-Smale model with processing delay, J. Math. Anal. Appl., 415 (2014), 53-61.

[18] S. Motsch and E. Tadmor: A New Model for Self-organized Dynamics and Its Flocking Behavior. J. Stat. Phys. 144 (2011).

[19] S. Motsch and E. Tadmor: Heterophilious Dynamics Enhances Consensus. SIAM review 56 (4), 577-621 (2014).

[20] S.-I. Niculescu: Delay Effects on Stability. A Robust Control Approach. Springer-Verlag London, 2001. 
[21] A. Seuret, V. Dimos, V. Dimarogonas and K.H. Johansson: Consensus under Communication Delays. Proceedings of the 47th IEEE Conference on Decision and Control, Cancun, Mexico, Dec. 9-11, 2008.

[22] H. Smith: An Introduction to Delay Differential Equations with Applications to the Life Sciences. Springer New York Dordrecht Heidelberg London, 2011.

[23] K. Szwaykowska, I.B. Schwartz, L.M. Romero, C.R. Heckman, D. Mox and M. Ani Hsieh: Collective motion patterns of swarms with delay coupling: theory and experiment. Phys. Rev. E 93, 032307.

[24] C. Somarakis and J. Baras: A Simple Proof of the Continuous Time Linear Consensus Problem with Applications in Non-Linear Flocking Networks. 2015 European Control Conference (ECC), 1546-1553.

[25] C. Somarakis and J. Baras: Delay-independent convergence for linear consensus networks with applications to non-linear flocking systems. In Proceedings of the 12th IFAC Workshop on Time Delay Systems, pp. 159-164, Ann Arbor (2015).

[26] C. Villani: Topics in optimal transportation. Graduate Studies in Mathematics 58 (2003), American Mathematical Society, Providence, RI. 